\title{
Important information
}

With the beginning of the first issue in 1999 Zeitschrift für Ernährungswissenschaft will undergo a relaunch. The name will be changed to European Journal of Nutrition and the Publication Language will be exclusively English.

Aims and Scope: The major focus of manuscripts submitted to the European Journal of Nutrition should consequently be on:

- cellular and molecular aspects of nutrition

- mechanistic studies on interactions between nutrients and non-nutrient food components on cell, organ, and body functions

- epidemiology with emphasis on the use of biomarkers

- nutrient metabolism in humans

- studies on the relation between individual genetic susceptibility, nutrition, and disease

- regulation of gene expression through nutrients or nonnutrient food components

Animal nutrition studies will only be considered for publication if a strong relation to actual problems in human nutrition is presented.

Members of the new Editorial Board are:

Christian A. Barth, Potsdam-Rehbrücke, D

barth@www.dife.de

Andrew R. Collins, Aberdeen, UK

a.Collins@rri.sari.ac.uk

Hannelore Daniel, Gießen, D

hannelore.daniel@ernaehrung.uni-giessen.de

Piero Dolara, Florence, I

dolara@stat.ds.unifi.it

Helmut F. Erbersdobler, Kiel, D

erbersdobler@nutrfoodsc.uni-kiel.de
Marc Ferrara, St.-Genes Champanelle, F

marc.ferrara@clermont.inra.fr

Frans J. Kok, Wageningen, NL

frans.kok@staff.nutepi.wau.nl

Miquel Moretó Pedragosa, Barcelona, E mmoreto@far.ub.es

Andrea Pfeifer, Vevey, $\mathrm{CH}$

andrea.pfeifer@chlsnr.nestrd.ch

Gerhard Rechkemmer, Karlsruhe, D

gerhard.rechkemmer@bfe.fzk.de

Peter Stehle, Bonn, D

p.stehle@uni-bonn.de

Gerhard Rechkemmer, Karlsruhe, D, will act as coordinating editor in the future.

Additionally an international Advisory Board will be established.

\section{Announcement to the authors:}

To enable an effective and fast publication process you can contact the editors by email to introduce your future contribution by a 2 page structured abstract as follows: Background - Aim of the study - Methods - Results Conclusions.

The editors are endeavored to answer within 3 or 4 days whether your contribution covers the Aims \& Scope.

Helmut F. Erbersdobler

Christian A. Barth 